\title{
Phase Diagram of Self-assembled Viral Capsid Protein Polymorphs ${ }^{\dagger}$
}

\author{
L. Lavelle, ${ }^{\ddagger}$ M. Gingery, ${ }^{\S}$ M. Phillips, ${ }^{\S}$ W. M. Gelbart, ${ }^{\ddagger}$ and C. M. Knobler*, \\ Department of Chemistry and Biochemistry, University of California, Los Angeles, \\ California 90095-1569, Molecular Biology Institute, University of California, Los Angeles, \\ California 90095-1570
}

\author{
R. D. Cadena-Nava,${ }^{\perp}$ J. R. Vega-Acosta,${ }^{\perp}$ L. A. Pinedo-Torres, and J. Ruiz-Garcia* \\ Instituto de Física, Universidad Autónoma de San Luis Potosí, Alvaro Obregón 64, \\ San Luis Potosí, S.L.P., 78000 México
}

Received: September 8, 2008; Revised Manuscript Received: November 12, 2008

\begin{abstract}
We present an experimental study of the self-assembly of capsid proteins of the cowpea chlorotic mosaic virus (CCMV), in the absence of the viral genome, as a function of $\mathrm{pH}$ and ionic strength. In accord with previous measurements, a wide range of polymorphs can be identified by electron microscopy, among them single and multiwalled shells and tubes. The images are analyzed with respect to size and shape of aggregates, and evidence is given that equilibrium has been achieved, allowing a phase diagram to be constructed. Some previously unreported structures are also described. The range and stability of the polymorphs can be understood in terms of electrostatic interactions and the way they affect the spontaneous curvature of protein networks and the relative stabilities of pentamers and hexamers.
\end{abstract}

\section{Introduction}

The simplest viruses consist of a single-protein-thick shell, the capsid, that surrounds and protects the viral genome. In tobacco mosaic virus (TMV), the capsid is a hollow cylinder, made up of many copies of a single protein, that assembles around its single-stranded RNA genome. In 1955 the demonstration by Fraenkel-Conrat and Williams ${ }^{1}$ that infectious TMV could spontaneously form in solutions of the pure capsid protein and RNA made it clear that the general physical principles of self-assembly could apply to a virus. A dozen years later, Bancroft and Hiebert ${ }^{2}$ showed that infectious cowpea chlorotic mosaic virus (CCMV), a spherical virus, would also spontaneously assemble from appropriately buffered solutions of its pure protein and RNA.

The physical basis of the assembly process has been made even more evident by the discovery that capsids could selfassemble around RNAs derived from different viruses and from nonviral anionic polymers ${ }^{3}$ or even gold nanoparticles. ${ }^{4}$ Moreover, under appropriate conditions, assembly of CCMV capsid protein can take place in the absence of RNA, leading to empty capsids and other structures such as multishells and tubes. The stability of such polymorphs is determined by the solution $\mathrm{pH}$ and ionic strength. ${ }^{3,5}$ Adolph and Butler" constructed the "phase diagram" for CCMV protein shown in Figure 1, which shows the range of stability of several structural forms as a function of the $\mathrm{pH}$ and ionic strength. A number of other polymorphs were identified by Bancroft and co-workers ${ }^{3}$ but they were obtained in experiments involving RNase, an enzyme that catalyzes the cutting of RNA. The intact virus was first brought to $\mathrm{pH} 7$ at low ionic strength, conditions at which the capsid

\footnotetext{
"Part of the "PGG (Pierre-Gilles de Gennes) Memorial Issue".

* Authors to whom correspondence should be addressed. E-mail: knobler@chem.ucla.edu (C.M.K.), jaime@ dec1.ifisica.uaslp.mx (J.R.-G.)

* Department of Chemistry and Biochemistry.

$\S$ Molecular Biology Institute.

$\perp$ These authors contributed equally to this work.
}

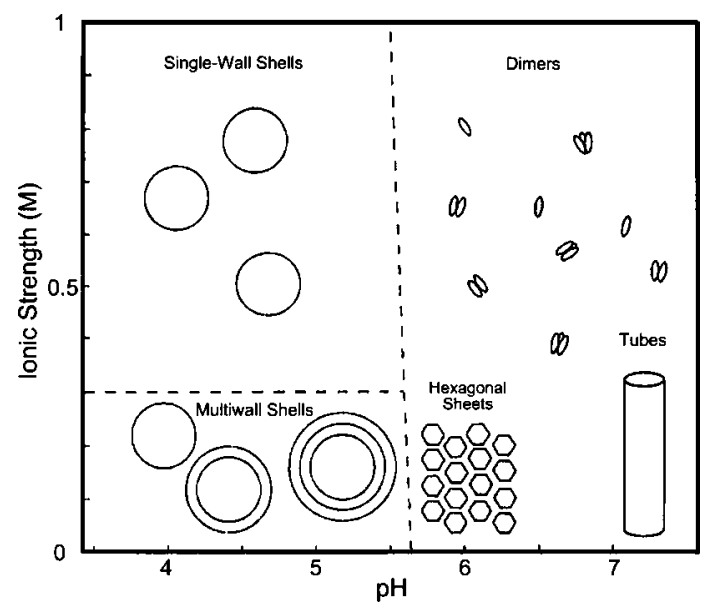

Figure 1. The CCMV capsid protein diagram adapted from Adolph and Butler. ${ }^{5}$ Shown are the conditions of $\mathrm{pH}$ and ionic strength at which the different types of polymorphs pictured are the dominant species.

undergoes a $10 \%$ radial expansion and no longer protects the genome from nuclease attack. The new polymorphs that form depend on the choice of nuclease, and they very likely contain RNA fragments; studies on self-assembly in alfalfa mosaic virus ${ }^{6}$ have demonstrated the sensitivity of viral protein structures to the length of the residual RNA.

The dependence of polymorph stability on $\mathrm{pH}$ and ionic strength is evidence that charge must play a significant role. The primary effect of the $\mathrm{pH}$ is to change the degree of ionization of the protein residues; the ionic strength primarily affects the range of the electrostatic interaction and the stabilizing effect of calcium ion binding to the capsid. But more general interactions between proteins and their effects on the spontaneous curvature and the relative stability of pentamers and hexamers must also play a role.

We investigate here the interplay between these factors by reexamining the CCMV protein phase diagram, identifying new 


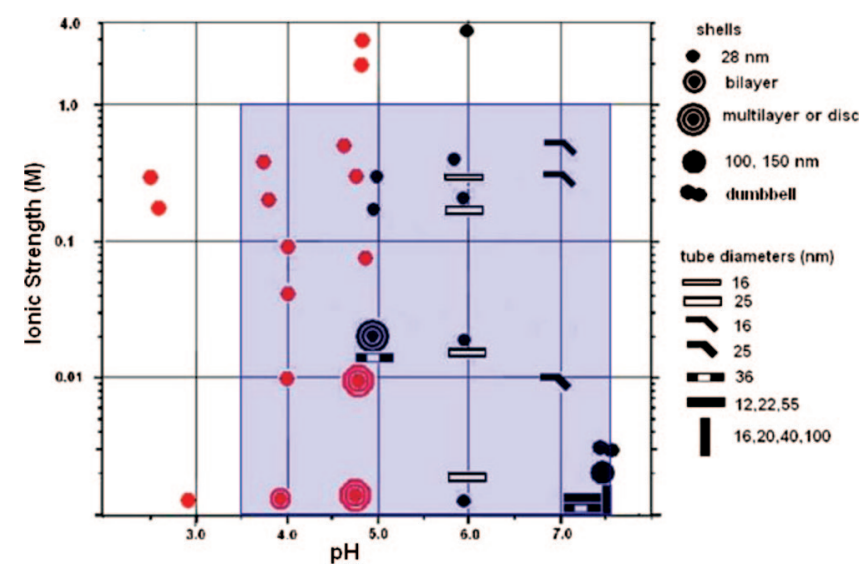

Figure 2. Phase diagram of the protein assembly as a function of $\mathrm{pH}$ and ionic strength, buffered with sodium cacodylate (red symbols) and sodium citrate (black symbols). (Although other buffers were used as well for preparing the samples, the results obtained here are consistent with those of these two buffers.) The blue rectangle indicates the conditions of assembly considered by Bancroft and co-workers ${ }^{3}$ and by Adolph and Butler; ${ }^{5}$ cf. Figure 1.

forms of aggregates, and focusing largely on the low-ionicstrength region where the range of structures is greatest. We interpret the diagram in terms of a recent theoretical treatment of viral protein assembly. ${ }^{7}$

\section{Experimental Results}

General Features of the Phase Diagram. Our phase diagram measurements have been carried out at over 100 conditions of $\mathrm{pH}$ and ionic strength $(I)$ using 7 buffers, covering the $\mathrm{pH}$ range $2.5-7.5$ and $I=0-4$. The protein concentrations were $0.1-0.5$ $\mathrm{mg} / \mathrm{ml}$, and the assembly was carried out at $4{ }^{\circ} \mathrm{C} ; \mathrm{NaCl}$ was used to adjust the ionic strength.

Figure 2 and Figure S1 in the Supporting Information show the conditions under which reassembly was examined and the buffers that were employed. For comparison, the conditions used by Adolph and Butler and Bancroft and co-workers are also shown. The most common structures are the single-walled shells, which are found over the range $\mathrm{pH}=2.5-7$ and $I=0.01-3$. It is only in the absence of added salt, where the ionic strength is controlled by the buffer concentration, that a large number of polymorphs are observed; accordingly, we will focus our attention on these low-ionic-strength conditions. As evident from the TEMs, and from previous studies, there is some heterogeneity in the structures, and the boundaries between stability regions are not sharp. In describing the polymorphs that characterize any particular region, we are referring to the dominant structures observed under those conditions.

At $\mathrm{pH}<3$, the protein assembles into single-wall spherical shells. Only a few such structures were found in each image at $I=0.2$, with the number increasing to a few tens at $I<0.01$, and a few hundreds above $\mathrm{pH}$ 3. Typical images under these experimental conditions are shown in Figure 3. There is no obvious dependence of the shell diameters on $\mathrm{pH}$ and ionic strength. A histogram constructed for 2500 single-wall shells, Figure 4, gives an average diameter of $28 \mathrm{~nm}$ and a half-width of $3 \mathrm{~nm}$. The dispersion in size is similar to that found in TEM measurements of intact viruses.

Two-layer shells begin to appear near $\mathrm{pH} 3.7$, and multiwall shells appear at about $\mathrm{pH}$ 4.7. Two- and three-wall structures are most frequently observed, but there are some four-wall shells and, rarely, shells with five walls; compare, Figure 5. The two- and three-wall shells are generally well formed, that is,

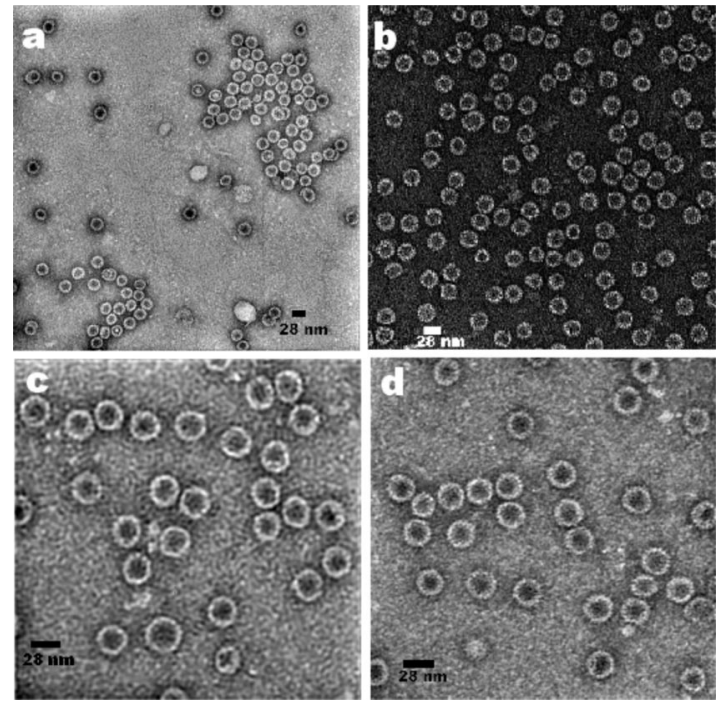

Figure 3. Typical images of single-wall shells. (a) $0.1 \mathrm{M}$ sodium acetate buffer, $\mathrm{pH}=4.08, I=3.0$; (b) $0.01 \mathrm{M}$ sodium acetate buffer, $\mathrm{pH}=4.0, I=0.01$; $(\mathrm{c}$ and $\mathrm{d}) 0.1 \mathrm{M}$ sodium acetate buffer, $\mathrm{pH}=$ $4.67, I=0.1$.

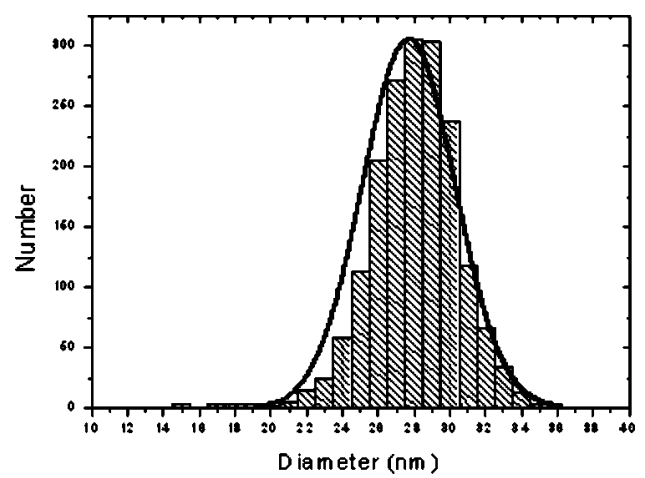

Figure 4. Diameter distribution of single-wall shells obtained at different $\mathrm{pH}$ and ionic strength. The line is a Gaussian fit to all data, and its maximum is located at $27.89 \mathrm{~nm}$.
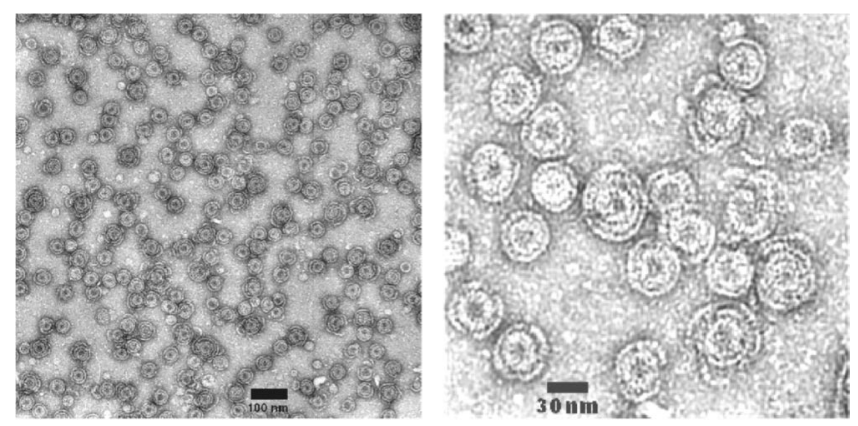

Figure 5. Typical images of multiwall shells obtained with sodium acetate buffer at (a) $\mathrm{pH}=4.8, I=0.10 \mathrm{M}$ and (b) $\mathrm{pH}=4.8, I=0.01$. Multiwall shells are the dominant structure as single-wall shells are scarce at these conditions.

rounded and complete. On the other hand, shells with four and five walls are often deformed and incomplete. As the ionic strength decreases, multiwall shells become the dominant structures. In general, the fraction of single-wall shells decreases with increasing $\mathrm{pH}$, and none are found above $\mathrm{pH} 7$.

At very low ionic strength occasional tubes are found in coexistence with shells at a $\mathrm{pH}$ as low as 3.8 , but the tubes become more numerous at $\mathrm{pH} 4$.1. At low $\mathrm{pH}$, tubes often have irregular diameters, see Figure $6 \mathrm{a}$, but they become more uniform and longer with increasing $\mathrm{pH}$, Figure $6 \mathrm{~b}$. Tubes at 

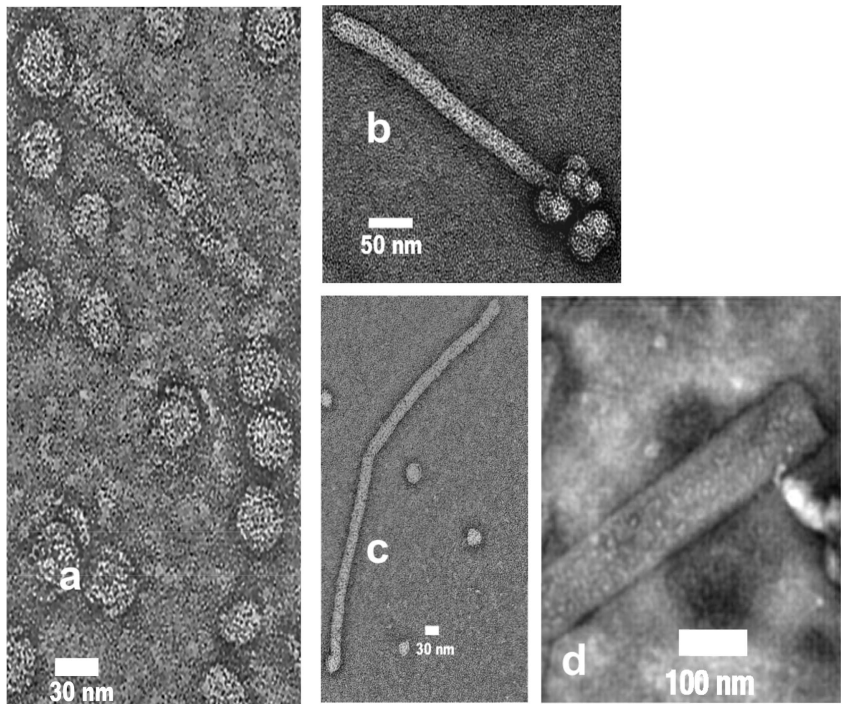

Figure 6. Tube structures obtained with sodium cacodylate buffer and no added salt: (a) $\mathrm{pH}=5.65,0.01 \mathrm{M}$ buffer; (b) $\mathrm{pH}=6.0,0.01 \mathrm{M}$ buffer; (c) $\mathrm{pH}=7.1,0.01 \mathrm{M}$ buffer; (d) $\mathrm{pH}=7.5,0.001 \mathrm{M}$ buffer.
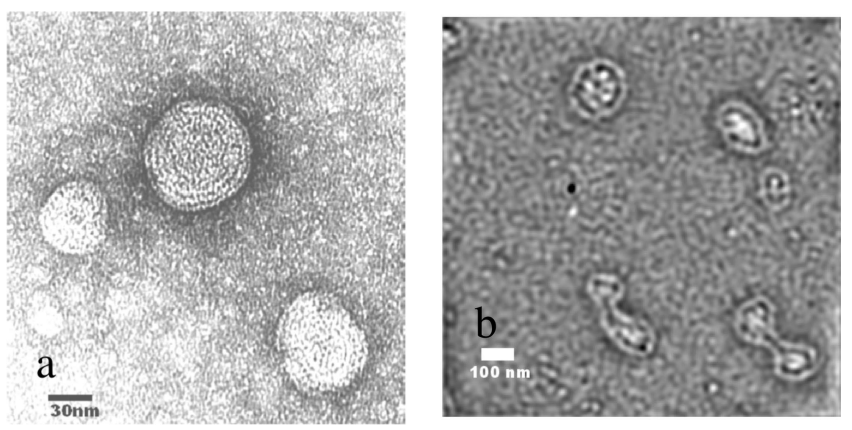

Figure 7. (a) Disks formed in $0.01 \mathrm{M}$ sodium citrate buffer $\mathrm{pH}=$ 4.75, no added salt. (b) Dumbbell structures at $\mathrm{pH}=7.5,0.001 \mathrm{M}$ cacodylate buffer, no added salt.

higher ionic strength tend to be narrower, $12-16 \mathrm{~nm}$ in average diameter; at low $\mathrm{pH}$ they are bent/kinked and their ends tend to be sharp, see Figure 6c. As the buffer concentration and ionic strength decrease, the tubes are better-formed and are wider, about $22-25 \mathrm{~nm}$ in diameter. Tube ends are rounded and can be bulbous, Figure 6, panels a and c; as in Figure 6b, they sometimes give the appearance of having grown out of a shell or a cluster of shells. Tube lengths range from 70 to $900 \mathrm{~nm}$. At higher $\mathrm{pH}$ values (>7.2) long, open-ended tubes, Figure $6 \mathrm{~d}$, with diameters as large as $100 \mathrm{~nm}$ are formed.

Table S1 in the Supporting Information summarizes the characteristics of the tubes observed with cacodylate buffer in this study. Very well formed tubes always appear at low ionic strength in a wide range of pHs. As we increase the ionic strength, the tubes become narrower and they tend to involve bends or kinks; some develop sharp or "spiky" ends.

Novel Structures. We have observed three novel structures that have not been previously reported. Disk-like structures such as those shown in Figure 7 a were observed at $\mathrm{pH}$ values ranging from 4.75 to 5.0 for ionic strengths up to 0.02 . Fourier transforms of the images show a hexagonal arrangement of the proteins that is expected for a lamellar structure. Although Bancroft et al. did not observe disks, they reported the formation of a large number of laminar and plate forms in the same range of conditions, thought to be formed on surfaces; the number of plate structures they observed is large compared with the number of disks we observed.

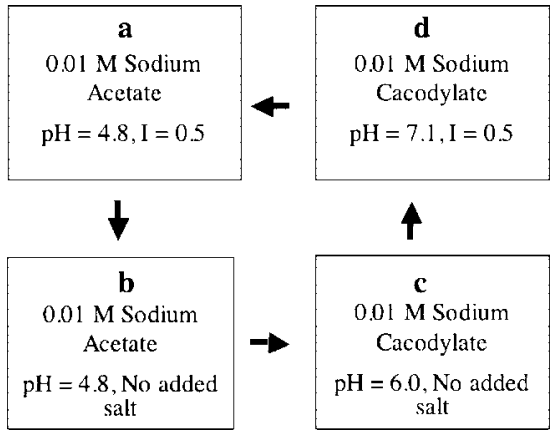

Figure 8. Path followed in test of thermodynamic equilibrium. The conditions are those given in each box and the arrows indicate dialysis for $24 \mathrm{~h}$.

At very low ionic strength and $\mathrm{pH}=7.5$ we found very large shells that are not well rounded and have 100-150 nm diameters; dumbbell-like shells, Figure $7 b$, were found under the same conditions.

Thermodynamic Stability. In ascribing a phase diagram to the polymorphs we are implicitly assuming that the structures are thermodynamically stable and are not kinetically controlled. Bancroft ${ }^{3}$ reported that CCMV protein dialyzed at $\mathrm{pH} 6.0$ and $0.5 \mathrm{M} \mathrm{NaCl}$ did not assemble. However, protein that was first dialyzed at $\mathrm{pH} 5.0$ and $0.5 \mathrm{M} \mathrm{NaCl}$ and then dialysed to $\mathrm{pH}$ 6.0 at the same ionic strength assembled into single-wall shells. Although it is not clear how long dialyses were carried out in this work, in some experiments by the Bancroft group ${ }^{8}$ it was noted that dialyses were carried out for 2-4 h. Adolph and Butler $^{5}$ argued that equilibrium could be attained by sufficiently long (48 h) dialysis. This is in accord with our observation that there was no significant difference between the results of dialysis for 24 or $120 \mathrm{~h}$.

As a test of thermodynamic stability we have carried out the four-step cycle shown in Figure 8, each time waiting $24 \mathrm{~h}$ after changing conditions, removing small aliquots, and determining the dominant structures in them by TEM. At the initial conditions ( $0.01 \mathrm{M}$ sodium acetate buffer, $\mathrm{pH} 4.8, I=0.5)$, the protein assembled into empty single-walled shells, Figure 9a. After dialysis at the same $\mathrm{pH}$ in the absence of added salt, multiwall shells formed, Figure 9b. Dialysis now against a 0.01 $\mathrm{M}$ sodium cacodylate buffer ( $\mathrm{pH}$ 5.95) led to reassembly into tubes, Figure 9c. When the system was then dialyzed to $\mathrm{pH}$ $7.12, I=0.5 \mathrm{M}$ with sodium cacodylate, the protein disassembled and no structures were observed, Figure 9d. Finally, when the system was dialysed to the initial conditions, single-walled shells were again obtained, Figure 9e. Particle size distributions at points a and e, as determined by light scattering, Table S2 in the Supporting Information, are essentially identical. We conclude then that the structures we have observed are representative of equilibrium.

Although the observed morphologies are controlled by solution conditions, defects in the structures, such as bends in tubes, are also observed. A striking example, the coil structures shown in Figure 10 are observed in a very narrow range of $\mathrm{pH}$, 4.7-4.8, and over a broad range of ionic strength, $\sim 0$ to 3.0 M. Plastic framework models of shells demonstrate that such structures can arise from an error in assembly in which a single pentamer is replaced by a hexamer. A variety of such misassembled structures - including dumbbells - have also been observed in molecular dynamics simulations of coarse-grained models of capsid proteins. ${ }^{9}$ These have been compared to socalled "monster" structures that were found in in vitro studies of the assembly of turnip crinkle virus and capsid protein around the viral RNA. ${ }^{10}$ 

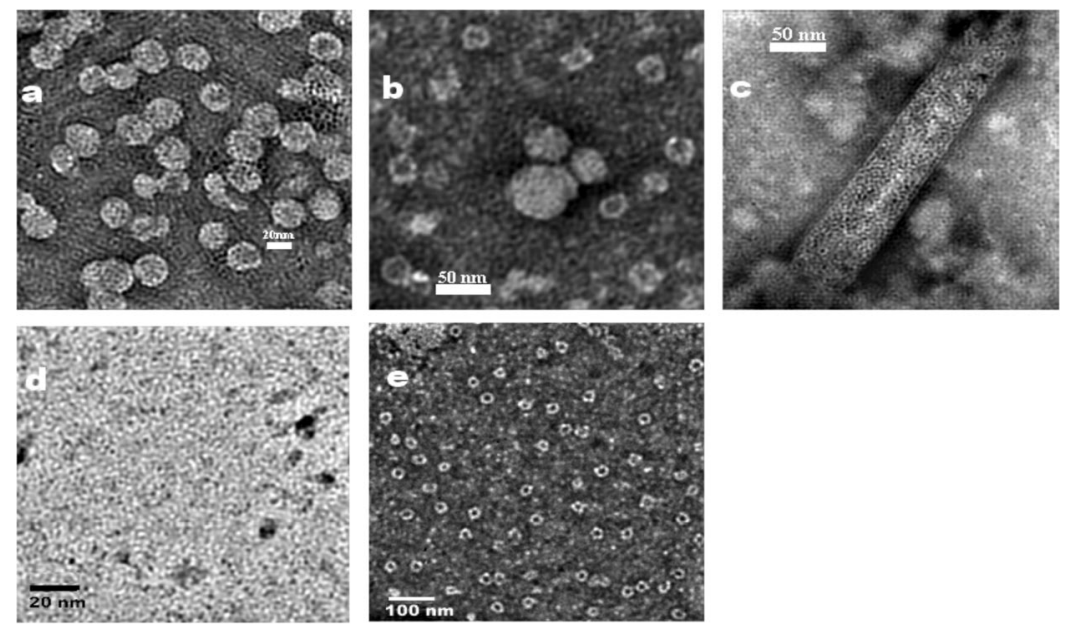

Figure 9. Images obtained at each step of the cycle shown in Figure 8. (a) Single-walled shell assemblies (28 nm). (b) Multishells (52 nm). (c) Tube (55 nm wide). (d) No structures were expected at these conditions. (e) Single-walled shells (28 nm).

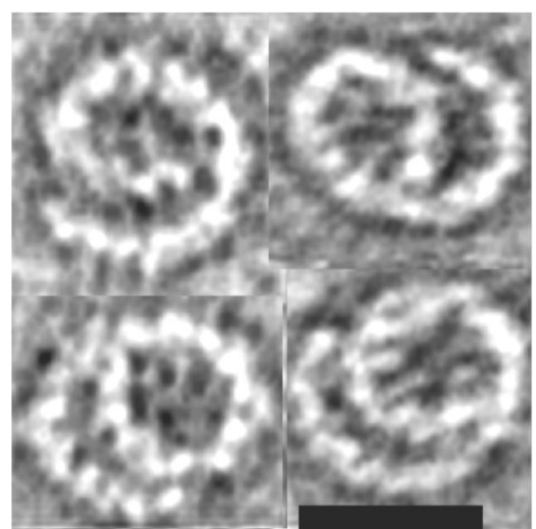

Figure 10. Coils found in coexistence with single-wall "normal" shells in $0.1 \mathrm{M}$ sodium acetate buffer, $\mathrm{pH}=4.8$. The upper two coils are with $I=2.0 \mathrm{M}$, the lower two at $I=3 \mathrm{M}$. The bar in the lower-righthand side image represents $28 \mathrm{~nm}$.

Quantitative Features of the Structures. Although capsids of the wild-type virus have diameters of $28 \mathrm{~nm}$, icosahedral capsids with smaller and larger diameters can also assemble. The capsids are characterized by their triangulation numbers, $T$, which were introduced by Caspar and $\mathrm{Klug}^{11}$ and are determined from the relation $T=h^{2}+k^{2}+h k$, with $h$ and $k$ equal to zero or a positive integer, resulting in $T$ numbers of 1 , $3,4,7,9,12 \ldots$ A capsid with triangulation number $T$ contains $60 T$ proteins, organized into 12 pentamer units and $10(T-1)$ hexamers. The various CCMV capsids observed to date ${ }^{12}$ correspond to Caspar-Klug structures with $T=1,3,4$, and 7; a so-called pseudo-Caspar-Klug structure with $T=2$ is also known. ${ }^{13}$ The relative diameters of the capsids are related to their $T$ numbers by the expression

$$
\frac{D_{i}}{D_{j}}=\sqrt{\frac{T_{i}}{T_{j}}}
$$

because the capsid surface area is proportional to $T$ (and $D^{2}$ ).

One might expect each of the concentric shells in multiwall assemblies to be related to a $T$ number with a diameter consistent with eq 1 . It is evident from the size distributions for complete triple-wall shells in Figure 11, however, that this is not the case. The average diameter for the innermost shell is $28.2 \pm 2.0 \mathrm{~nm}$, in good accord with the diameters of single-wall, $T=3$ shells.
The average diameter of the second shell is $39.5 \pm 1.9 \mathrm{~nm}$, markedly larger than the $32 \mathrm{~nm}$ expected for a $T=4$ structure and smaller than the $43 \mathrm{~nm}$ expected for $T=7$. Similarly, the average outer-shell diameter, $51.8 \pm 3.0$, is larger than the 48 $\mathrm{nm}$ expected for a shell with $T=9$ and smaller than the $56 \mathrm{~nm}$ expected for a $T=12$ capsid.

These results demonstrate that the successive shells do not have a regular icosahedral structure and are disordered, in accord with recent studies of the assembly of CCMV protein around nanoemulsion droplets of different sizes in which disordered structures were characterized. ${ }^{14}$ This differs from the case of double-shell viral capsids such as those found in Bluetongue virus ${ }^{15}$ in which a $T=13$ shell surrounds a $T=2$ shell, or rhesus rotavirus, ${ }^{16}$ in which both the inner and outer shells have $T=13$ triangulation numbers. In these viruses the proteins in the inner and outer shells differ, and there are connections between the shells that maintain the separation and orientation.

\section{Discussion}

The role of charge and spontaneous curvature on the selfassembly of amphiphiles into structures such as unilamellar and multilamellar spherical and tubular vesicles is well understood. ${ }^{17}$ These principles can be applied as well to the assembly of viral capsid protein; in addition, however, specifics of packing of the protein into hexamers and pentamers must be taken into consideration. The charge is determined by the degree of ionization of the acidic and basic amino acid residues, which is controlled by the $\mathrm{pH}$, and the range of the Coulombic interaction is controlled by the ionic strength. Accordingly, $\mathrm{pH}$ and ionic strength are natural variables for the phase diagram. The relative stabilities of hexamers and pentamers, the energy difference between them, is another determining factor, but this, in turn can be related to the $\mathrm{pH}$.

The physical considerations underlying the assembly and stability of single-wall shells and the effect of $\mathrm{pH}$ have already been described in detail. ${ }^{18,19}$ There has been only one theoretical treatment of the factors that control the stability of multishells and tubes, ${ }^{7}$ and we use it as a basis for the qualitative understanding of the phase diagram. A key to an understanding of multishell stability is the $\mathrm{pH}$ dependence of the single-wall shell, as shown by the electrophoretic mobility. Johnson, et al. ${ }^{20}$ have measured the mobilities of the virus and of empty capsids; their results are shown in Figure 12. They explained the small difference in the mobilites between the empty and full shells 

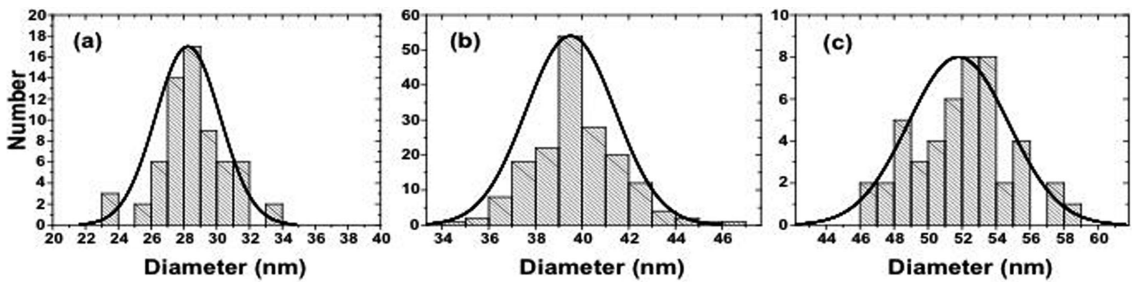

Figure 11. Diameter distributions of the (a) innermost, (b) middle, and (c) outermost shells of triple-wall shells.

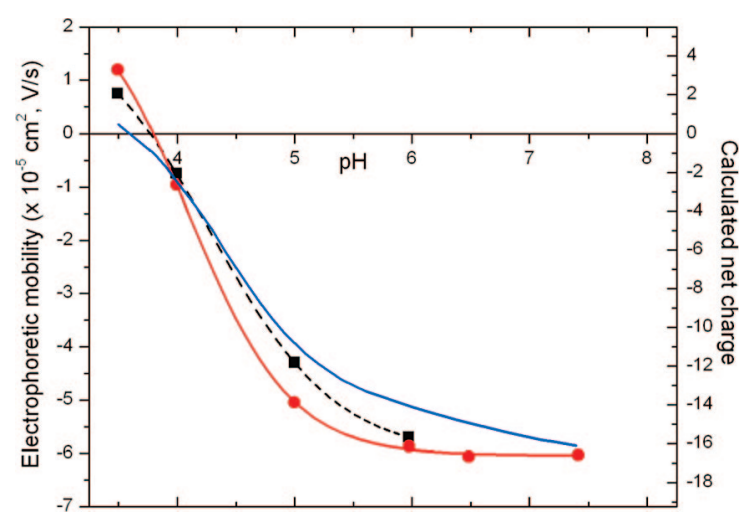

Figure 12. Electrophoretic mobility of CCMV capsids as a function of $\mathrm{pH}$. The full red line is the mobility for the intact virus, and the dashed line is that for empty capids (single-walled shells) measured by Johnson, et al. ${ }^{20}$ in the presence of $0.2 \mathrm{M} \mathrm{NaCl}$. The net charge, represented by the blue line, has been calculated from the $\mathrm{p} K_{\mathrm{a}}$ values of the isolated amino acid residues of the capsid protein with the omission of the 12 lysine and 9 arginine residues. To compare the measured mobilities and the calculated net charge, the right-hand ordinate has been multiplied by an arbitrary scale factor.

as the result of the dependence of the mobility on the surface charge density rather than on the net charge.

In CCMV, the surface charge arises from the acidic amino acid residues, which lie predominantly on the capsid exterior. In contrast, most of the basic residues are near the amino terminus and project into the capsid interior. ${ }^{21} \mathrm{~A}$ simple calculation of the $\mathrm{pH}$ dependence of the charge on a CCMV capsid on the $\mathrm{pH}$ from the $\mathrm{p} K_{\mathrm{a}}$ values of the isolated residues in which the contribution of the basic (largely interior) lysine and arginine residues is omitted - gives an isoelectric point of 3.6, which is essentially identical to that of CCMV. Moreover, as shown in Figure 12, the shape of the curve is closely similar to the measured electrophoretic mobility. These results are consistent with the argument that the electrophoretic mobility is associated with the surface charge density.

The electrophoretic mobility measurements in Figure 12 were carried out only at $0.2 \mathrm{M} \mathrm{NaCl}$. However, recent studies on the spherical bacteriophage MS2 and accompanying theory ${ }^{22}$ show that for a layered structure such as a virus the magnitude of the electrophoretic mobility depends on the ionic strength but the isoelectric point shows little change.

The net charge on the capsid is determined by the balance between the charges of the acidic residues, which lie predominantly on the exterior of the capsid, and the basic residues, lysine and arginine, have $\mathrm{p} K_{\mathrm{a}}$ values in excess of 10 and are therefore charged over the entire $\mathrm{pH}$ range that has been examined in the assembly reactions. The $\mathrm{pKa}$ values of the acidic residues, aspartic acid and glutamic acid, are much lower, and their contribution to the charge therefore varies at acidic $\mathrm{pH}$. (Note that the $\mathrm{p} K_{\mathrm{a}}$ values are not those of the isolated residues and are dependent on the local environment. ${ }^{23}$ )

We have observed that multishell formation begins near $\mathrm{pH}$ 3.7, which is the isoelectric point of empty capsids (Figure 12), that is, single-wall shells. This is related to the fact that, as the $\mathrm{pH}$ increases, the capsid exterior becomes increasingly negatively charged because of the ionization of acidic residues. The addition of a second shell around the capsid is then favored because of the electrostatic interaction between the exterior negative surface charge and the positive charge on the proteins at the interior surface. In the related case of oppositely charged flat membranes ${ }^{24}$ there is a stable equilibrium separation that is determined by the difference in the charge densities and the counterion concentration. Here, however, there is an additional feature, the spontaneous curvature of the protein shell, which prefers a radius of $14 \mathrm{~nm}$. The successive shells must have larger radii of curvature, and the energy cost for the bending away from the spontaneous curvature will also play a role in controlling the spacing. With increasing $\mathrm{pH}$ the charge difference between the interior and exterior surfaces becomes larger, favoring the formation of more shells, but at the same time the shell curvature must be decreased and the energy penalty grows. Multishell stability will decrease with increasing ionic strength because the screening of charge reduces the strength of the Coulombic interaction.

Implicit in these arguments about the effect of the spontaneous curvature is the assumption that the interactions between capsid proteins that determine the curvature are not significantly affected by the $\mathrm{pH}$. It is known ${ }^{21}$ that the diameter of CCMV capsids does not change until the $\mathrm{pH}$ is close to 7 , at which point the capsid diameter expands by $10 \%$. Thus, the interprotein interactions are not affected in the range of $\mathrm{pH}$ at which multishells are stable. The curvature may well change, however, if the capsid structure is disordered, which appears to be the case for successive shells. This would have a quantitative effect on the curvature but is unlikely to affect the qualitative features of the competing interactions.

The leveling out of the electrophoretic mobility near $\mathrm{pH}$, as shown in Figure 12, limits the strength of the electrostatic interaction between shells; additional layers cannot grow because the curvature energy cannot be compensated. This is seen in the structures (called "rosettes" by Bancroft) shown in Figure 13, in which incomplete shells surround a multishell. In general, the rosette "petals" (see arrows) have a higher curvature than the last complete shell and therefore bend out.

The transition from spherical to tubular capsids is related to the difference in energy between hexamers and pentamers of proteins. Crudely speaking, this difference arises because pentamers have five interactions with their neighbors rather than six. The energy of a capsid then depends on the hexamerpentamer energy penalty, which increases with increasing $\mathrm{pH}^{25}$ As shown by Bruinsma, et al., ${ }^{7}$ if the penalty is sufficiently large (i.e., at a sufficiently high $\mathrm{pH}$ ) an assembly of spherical capsids becomes unstable with respect to spherocylindrical capsids with the same mean curvature and the same total area.

Using optical Fourier transforms of electron micrographs, Bancroft et al. ${ }^{8}$ showed that CCMV tubes have a hexagonal structure. Tubes with rounded ends are composed of a hexagonal 


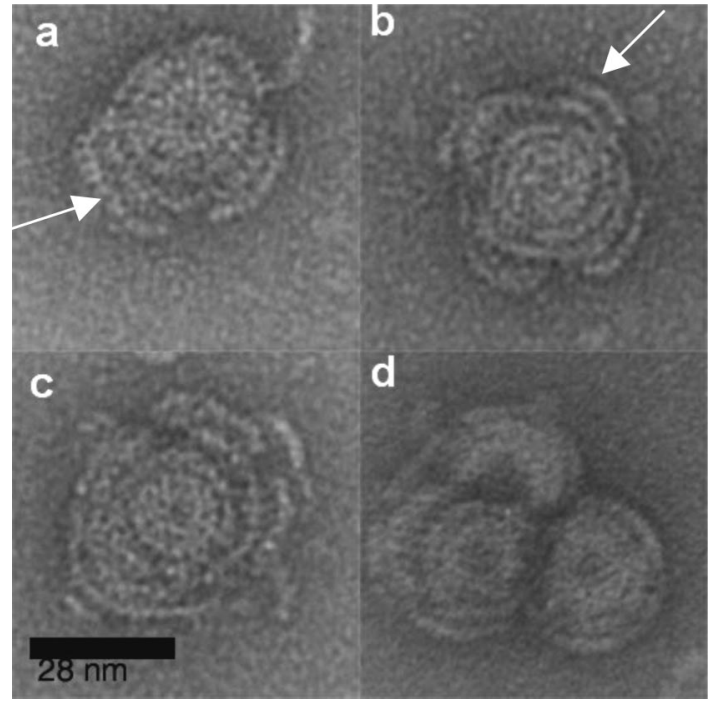

Figure 13. Rosette structures in which incomplete shells form petals (see arrows in panels a and b) with different curvature surrounding a completed shell.

sheet rolled into a cylinder and capped by hemispheres composed of pentamers and hexamers. The tube diameters can be understood in terms of a balance between the curvature of the caps, which depends on the hexamer-pentamer penalty, and the spontaneous curvature of a sheet composed of hexamers, which is related to the charge asymmetry between the two sides. We expect discrete diameters because, like complete capsids, hemispherical caps can exist only for specific $T$ numbers and because the joining of sheets to form a tube requires that there be a continuous hexagonal structure.

In their seminal paper on the symmetry of viral capsids, Caspar and Klug ${ }^{11}$ suggested that one of the most probable "mistakes" of assembly was a tube produced by rolling a hexagonal plane lattice into a tube. If the tubes were capped by icosahedra from pentamers and hexamers they would have diameters comparable to those of fully assembled capsids. As first pointed out by Bancroft, ${ }^{3}$ the majority of the tubes fall into two classes: narrow tubes with diameters about $16 \mathrm{~nm}$ and wide tubes about $25 \mathrm{~nm}$ in diameter. The small diameter corresponds to that of a $T=1 \mathrm{cap}$, which from eq 1 is $16 \mathrm{~nm}$ (based on $D$ $=28 \mathrm{~nm}$ for $T=3$ ), and the larger diameter to a $T=2$ cap, which has a $24 \mathrm{~nm}$ diameter. The smaller diameter is favored at higher ionic strength, where the electrostatic screening reduces the spontaneous curvature of the hexagonal sheet by reducing the effect of the inner surface-outer surface charge asymmetry. The diameters of the structures of the uncapped tubes are determined by the curvature of the hexagonal sheet and by the requirement that the hexagonal structure be continuous.

Kieslev and $\mathrm{Klug}^{26}$ also found small and large diameter tubes for the capsid protein of rabbit papilloma virus. Although this virus has $T=7$ capsids, the larger tubes correspond to $T=3$ caps and the smaller to $T=1$. Fourier transforms of the electron micrographs showed that the larger tubes are hexagonal. The smaller tubes, which are capped by six pentamers, have an unusual structure in which pentamers are organized as hexavalent units, allowing the tube surface to be tiled. Our measurements do not allow us to distinguish between this structure and one in which a hexagonal sheet of hexamers is capped by six pentamers.

When the energy penalty for pentamers becomes sufficiently large, the spherical caps will be lost, leaving tubes with straight ends. (There is, of course, an energy penalty for the unbonded hexamers at the ends, so strictly speaking it is the difference between it and the cost for pentamers that controls the uncapping.) The diameters of the tubes then depend only on the spontaneous curvature of the hexagonal sheet, which, as already pointed out, decreases with increasing salt.

Although we have discussed the origin of the most common of the polymorphs, still others are found in small quantities, underlining the richness of the viral protein assemblies. The sensitivity to charge suggests that still other structures might be found if the assembly was carried out with protein mutants. A variety of polymorphs has been found for a number of different viral proteins and the arguments that we have made about the factors that determine CCMV assemblies should apply broadly. It should be noted as well that beside $\mathrm{pH}$ and ionic strength, the relative stability of hexamers and pentamers may be affected by the presence of small molecules that can bind to the capsid proteins. This is the mechanism proposed by Stray et al. ${ }^{27}$ for the formation of hexagonal sheets and tubes from hepatitis B viral proteins. In general, polymorphs of viral capsid proteins have little direct impact on our understanding of the biological function of viruses, but they may provide an opportunity for materials science applications, some of which have been explored for single-wall capsids. ${ }^{28}$

\section{Experimental Details}

CCMV was harvested from cowpea california black eye No. 5 plants infected both in Los Angeles and San Luis Potosi and purified by ultrafiltration using the detailed procedures developed and described by Michel, et al. ${ }^{29}$ (see Sections 2.1, 2.2 and 2.4).

The purified CCMV was disassembled, and the capsid protein was isolated using the detailed methods described by Lavelle et al. $^{30}$ (see Section 2.4). Protein purity and integrity were determined by SDS-PAGE and MALDI-TOF mass spectrometry. In this study, typical UV-absorbance ratios $\left(\mathrm{Abs}_{280 \mathrm{~nm}}\right.$ / $\left.\mathrm{Abs}_{260 \mathrm{~nm}}\right)$ were between 1.5 ( $0.5 \%$ nucleic acid contamination) and 1.6 ( $0.25 \%$ nucleic acid contamination). Protein concentrations were determined from the absorbance at $280 \mathrm{~nm}$ as previously described, ${ }^{30}$ and the reassembly was carried out at concentrations of $0.1-0.5 \mathrm{mg} \mathrm{ml}^{-1}$.

Reassembly of the CCMV protein capsid was as previously described $^{30}$ (see Section 2.5). However we note the following. The disassembly buffer $(0.9 \mathrm{M} \mathrm{NaCl}, 0.02 \mathrm{M}$ Tris- $\mathrm{HCl}, \mathrm{pH}$ 7.4, $1 \mathrm{mM}$ DTT, $0.5 \mathrm{mM}$ PMSF) contains DDT (dithiothreitol or Clealand's reagent) to prevent the formation of disulfide bonds and PMSF (phenylmethylsulfonylfluoride) to inhibit proteases. Prior to the reassembly experiments discussed in this paper, the DTT and PMSF were removed from the protein sample by centrifugation at $4{ }^{\circ} \mathrm{C}$ using a Centriplus YM-3 filter by dilution of the protein sample and centrifugation (three times) using disassembly buffer without DDT and PMSF.

The assembly reactions are carried out by dialyzing the protein in 200 to $300 \mu \mathrm{L}$ of the disassembly protein buffer solution, without PMSF and DTT in a $0.5 \mathrm{~mL}$ dialysis cassette (Pierce Slide-A-Lyser, $3.5 \mathrm{kDa}$ membrane) against a large excess of buffer (typically $0.5 \mathrm{~L}$ ) at a given ionic strength and $\mathrm{pH}$ for $24 \mathrm{~h}$ at $4{ }^{\circ} \mathrm{C}$. $\mathrm{NaCl}$ was used to adjust the ionic strength. Figure $\mathrm{S} 1$ of the Supporting Information shows the buffers used in this work, the $\mathrm{pH}$ range at which they were used, and a comparison with those used by Bancroft and co-workers ${ }^{3}$ and by Adolph and Butler. 5

Aliquots of the samples were deposited on grids made hydrophilic by glow discharge and were stained with uranyl acetate $(1 \% \mathrm{w} / \mathrm{v})$. Images were obtained with a Hitachi $\mathrm{H}-7000$ TEM (UCLA) and JEOL TEMs (JEM-1010 and JEM-1230) 
(UASLP) and enhanced by Fourier transformation with the program Digital Micrograph (Gatan Inc., Pleasanton, CA), application of a mask, and inverse Fourier transformation. The intensity and contrast were adjusted to optimize the image quality. The diameter of spherical particles was obtained by taking the geometric mean of at least two orthogonal measurements. To construct the particle size distribution histograms of the shell capsids, about 2000 particles were measured, whereas for the tubes several measurements of the diameter were taken along their length. A binning interval of $1 \mathrm{~nm}$ was used to construct each of the size distribution histograms.

Acknowledgment. The work described in this paper was supported by the U.S. National Science Foundation (Grants CHE 0400363 and CHE 0714411, to W.M.G. and C.M.K.), CONACYT (Grant 60833 to J.R.G.) and a UC MEXUS-CONACYT Collaborative Grant (to J.R.G., W.M.G., and C.M.K.). We thank Professor Joseph Loo for his assistance in carrying out the mass spectrometric measurements of the protein.

Supporting Information Available: This material is available free of charge via the Internet at http://pubs.acs.org.

\section{References and Notes}

(1) Fraenkel-Conrat, H.; Williams, C. Proc. Nat. Acad. Sci. USA 1955, $41,690-4$.

(2) Bancroft, J. B.; Hiebert, E. Virology 1967, 32, 354-6.

(3) Bancroft, J. B. Adv. Virus Res. 1970, 16, 99-134.

(4) See, e.g., Chen, C.; Daniel, M. C.; Quinkert, Z. T.; De, M.; Stein, B.; Bowman, V. D.; Chipman, P. R.; Rotello, V. M.; Kao, C. C.; Dragnea, B. Nano Lett. 2006, 6, 611-5.

(5) Adolph, K. W.; Butler, P. J. G. J. Mol. Biol. 1974, 88, 327-41.

(6) Lebeurier, G.; Fraenkel-Conrat, H.; Wurtz, M.; Hirth, L. Virology 1971, 43, 51-61.

(7) Bruinsma, R. F.; Gelbart, W. M.; Reguera, D.; Rudnick, J.; Zandi, R. Phys. Rev. Lett. 2003, 90, 248101. 379.

(8) Bancroft, J. B.; Hills, G. J.; Markham, R. Virology 1967, 31, 354-

(9) Nguyen, H. D.; Reddy, V. S.; Brooks III, C. L. Nano Lett. 2007, 7, 338-44.

(10) Sorger, P. K.; Stockley, P. G.; Harrison, S. C. J. Mol. Biol. 1986, $191,639-58$

(11) Caspar, D. L. D.; Klug, A. Cold Spring Harbor Symp. Quant. Biol. 1962, 27, 1-24.

(12) Hu, Y.; Zandi, R.; Anavitarte, A.; Knobler, C. M.; Gelbart, W. M. Biophys. J. 2008, 94, 1428-36.

(13) Tang, J.; Johnson, J. M.; Dryden, K. A.; Young, M. J.; Zlotnick, A.; Johnson, J. E. J. Struct. Biol. 2006, 154, 59-67.

(14) Chang, C. B.; Knobler, C. M.; Gelbart, W. M.; Mason, T. G. ACS Nano 2008, 2, 281-6.

(15) Grimes, J. M.; Burroughs, J. N.; Gouet, P.; Diprose, J. M.; Malby, R.; Ziéntra, S.; Mertens, P. P. C.; Stuart, D. I. Nature 1998, 395, 470-8.

(16) Yeager, M.; Dryden, K. A.; Olson, N. H.; Greenberg, H. B.; Baker, T. S. J. Cell Biol. 1990, 110, 2133-44.

(17) See, e.g., Gelbart, W. M.; Ben-Shaul, A. J. Phys. Chem. B 1996, 100, 13169-89.

(18) Kegel, W. K.; van der Schoot, P. Biophys. J. 2004, 86, 3905-13.

(19) Siber, A.; Podgornick, R. Phys. Rev. E 2007, 76, 061906.

(20) Johnson, M. W.; Wagner, G. W.; Bancroft, J. B. J. Gen. Virol. 1973, 19, 263-73.

(21) Speir, J. A.; Munshi, S.; Wang, G.; Baker, T. S.; Johnson, J. E. Structure 1995, 3, 63-77.

(22) Langlet, J.; Gaboriaud, F.; Gantzer, C.; Duval, J. F. L. Biophys. J. 2008, 94, 3293-312.

(23) Tama, F.; Brooks III, C. L. J. Mol. Biol. 2002, 318, 733-67.

(24) Ben Yaakov, D.; Burak, Y.; Andelman, D.; Safran, S. A. EPL 2007, 79,48002 .

(25) Johnson, J.; Speir, J. A J. Mol. Biol, 1997, 269, 665-75.

(26) Kiselev, N. A.; Klug, A. J. Mol. Biol. 1969, 40, 155-71.

(27) Stray, S, J.; Bourne, C. R.; Punna, S.; Lewis, W. G.; Finn, M. G.; Zlotnick, A. Proc. Natl. Acad. Sci. USA 2005, 102, 8138-8143.

(28) See, e.g Douglas, T.; Young, M. Adv. Mater. 1999, 11, 679-81.

(29) Michel, J. P.; Gingery, M.; Lavelle, L. J. Virol. Methods 2004, $122,195-8$.

(30) Lavelle, L.; Michel, J. P.; Gingery, M. J. Virol. Methods 2007, 146, 311-16.

JP8079765 